\title{
Diffusion of particles in an expanding sphere with an absorbing boundary
}

\author{
K. Forsberg ${ }^{1}$ and A. R. Massih ${ }^{2,3}+\underset{\dagger}{1}$ \\ ${ }^{1}$ Bronsåldersg. 6, SE-723 51 Västerås, Sweden \\ ${ }^{2}$ Quantum Technologies, Uppsala Science Park, SE-751 83 Uppsala and \\ ${ }^{3}$ Malmö University, SE-205 06 Malmö, Sweden \\ E-mail: alma@quantumtech.se
}

\begin{abstract}
We study the problem of particles undergoing Brownian motion in an expanding sphere whose surface is an absorbing boundary for the particles. The problem is akin to that of the diffusion of impurities in a grain of polycrystalline material undergoing grain growth. We solve the time dependent diffusion equation for particles in a $d$-dimensional expanding sphere to obtain the particle density function (function of space and time). The radius of sphere expands as $R=(K t)^{\alpha}$, where $\alpha$ is the growth exponent, $K$ a constant and $t$ the time. This allows the calculation of the survival rate or the total number of particles per unit volume as a function of time. We have obtained particular solutions exactly for the case where $d=3$ and a parabolic expansion of the sphere. Asymptotic solutions for the particle density when the sphere expansion rate is small relative to particle diffusivity and vice versa are derived.
\end{abstract}

$\ddagger$ Corresponding author. 


\section{Introduction}

The kinetics of many physical systems can be described by first-passage properties of stochastic systems (Redner 2001). In particular, the problem of the survivability of a diffusing particle, or Brownian walker, in a confined domain ("cage") with moving and absorbing boundary ("fence") has been a subject of several recent studies (Krapivsky \& Redner 1996, Redner 2001, Bray \& Smith 2007b, Bray \& Smith 2007a).

Krapivsky \& Redner (1996) evaluated the survival probability of a diffusing particle $S(t)$ as a function of time $t$ within a 1-dimensional expanding cage of length $L(t)$ with absorbing boundaries by solving the standard diffusion equation (heat equation) in one dimension. Approximate methods were devised in the limit of slow (adiabatic approximation) and fast (free approximation) motion of the absorbing boundary. More specifically, Krapivsky \& Redner (1996) considered $L(t) \sim t^{\alpha}$ with $\alpha<1 / 2$ for adiabatic approximation and $\alpha>1 / 2$ for free approximation. Moreover, the case of $\alpha=1 / 2$, corresponding to marginally expanding cage, was theoretically assessed, see also Redner (2001).

Bray \& Smith (2007b) calculated the exact asymptotic survival probability of a 1dimensional Brownian particle, initially confined at a position $x \in(-L(t), L(t))$ in the presence of two moving and absorbing boundaries by solving a Fokker-Planck equation for the system in the limit $t \rightarrow \infty$. Subsequently, they (Bray \& Smith 2007a) extended their method to treat the case of the $d$-dimensional system and in particular $d=3$. Similarly, in the latter work, they considered the steady-state case $(t \rightarrow \infty)$ and assumed the absorbing sphere's size evolves as $L(t)=L_{0}+v t$, where $L_{0}$ is the initial size and $v$ is the constant velocity of the boundary.

The aforementioned deliberations have relevance to the problem of diffusing

particles (impurities) in a grain of polycrystalline material under grain growth (Forsberg \& Massih 2007). Grain growth is a kinetic process at which the mean grain size of an aggregate of crystals increases during annealing at an elevated high temperature (Atkinson 1988). In this course, the larger grains are apt to increase in size at the expense of smaller grains which collapse, causing the total number of grains to decrease. Impurities in solid solution, prevalent in polycrystalline materials, which usually undergo diffusive motion at elevated temperatures, are in addition subjected to a moving or stretching medium due to grain growth. In particular, the process occurs in nuclear fuel (e.g., $\mathrm{UO}_{2}$ ), whereupon the fission product gases (e.g., Xe and $\mathrm{Kr}$ ) diffusing in the grain can be subjected to a moving grain boundary. Grain boundary movement can sweep up fission gas atoms more rapidly than they could have arrived at the boundary by diffusion (Hargreaves \& Collins 1976). This problem has been treated in (Forsberg \& Massih 2007). Modelling grain growth phenomenon or evolution of cellular structure in general has been a subject of many recent investigations using various theoretical and numerical approaches, see review by Thompson (2001). For example, in (Niemiec et al. 1998, Gadomski et al. 2003) a different approach is used to describe the grain growth process and for which the exponents of the growth asymptotes are presented. 
In this note, we solve the time-dependent diffusion equation for particles in a $d$ dimensional expanding spherical grain to obtain the particle density as a function of space and time. Equations of motion are treated in section 2, General solutions for particle density are provided in section 3. Special solutions are derived in section 4 for the case of $d=3$ and a parabolic grain growth. An evaluation of particle survival is presented in section 5. here we also include asymptotic solutions for particle density when grain growth rate is small relative to particle diffusivity and vice versa. We conclude the note in section 6 with a brief summary of the results.

\section{Equations of motion}

We consider the equivalent sphere model for material, meaning that the grains of the material are treated as a collection of spheres of uniform size characterized by a single equivalent radius, $R \equiv R(t)$, which is a function of time $t$. The particles (e.g. interstitials, impurities etc) migrate to grain boundaries by diffusion upon which are released from the system. The grain can contain traps (vacancies, pores, etc), which may capture the diffusing particle during its flight. The differential equation for the density of particles at position $r$ in a $d$-dimensional spherical grain at time $t, C(r, t)$, is given by the law of isotropic diffusion, viz.

$$
\frac{\partial C(r, t)}{\partial t}=D \nabla_{r}^{2} C(r, t),
$$

subject to the conditions,

$$
\begin{aligned}
& \frac{\partial C(0, t)}{\partial r}=0, \\
& C(R(t), t)=0, \\
& C(r, 0) \quad=C_{0},
\end{aligned}
$$

where $D$ is the effective diffusivity of particles in the grain, accounting for the presence of traps, $\nabla_{r}^{2}=\partial^{2} / \partial r^{2}+((d-1) / r) \partial / \partial r, C_{0}$ is the initial density of particles in the grain, and equation (3) defines the absorbing boundary (Dirichlet boundary condition). We should note that $D$, the effective diffusion coefficient of the particles in the grain, accounts for the effect of intragranular traps. As has been shown by Speight (1969), for stationary traps, it is related to the intrinsic diffusivity $D_{\text {int }}$ of particle in the solid through $D=p D_{\text {int }} /(p+g)$, where $p$ is the escape probability of the trapped particle and $g$ is the corresponding capture probability, related to trap size, the number of traps $n$ and the intrinsic diffusivity, e.g. for spherical traps, $g=4 \pi D_{\text {int }} \varrho n$, where $\varrho$ is the trap mean radius (Ham 1958). Thus at any time, only a fraction of the particles is trapped, while the rest are able to diffuse out of the grain.

The total number of particles per unit volume of $d$-dimensional spherical grain $\left(V_{d}\right)$ at time $t, G(t)$, is given by

$$
G(t)=\frac{d}{R^{d}(t)} \int_{0}^{R} r^{d-1} C(r, t) d r .
$$


The total number of particles contained in the grain at time $t$ is simply $S(t) \equiv V_{d}(t) G(t)$ with $V_{d}(t)=\pi^{d / 2} R^{d}(t) / \Gamma(d / 2+1)$ being the volume of sphere at time $t$; while the total number of particles released from the grain is

$$
F(t)=V_{d}(0) C_{0}-S(t) .
$$

In general, we allow the particle diffusion and grain growth to occur simultaneously; hence we scale equation (11) to embed the time-dependent variable $R(t)$ in the coordinate of the partial differential equation (space-time), namely

$$
\frac{\partial \mathcal{C}(\rho, \tau)}{\partial \tau}=\frac{\partial^{2} \mathcal{C}(\rho, \tau)}{\partial \rho^{2}}+\left[\frac{d-1}{\rho}+\frac{R_{\tau}}{R} \rho\right] \frac{\partial \mathcal{C}(\rho, \tau)}{\partial \rho}
$$

where we put

$$
\begin{aligned}
& \rho=\frac{r}{R}, \\
& \tau=D \int_{0}^{t} \frac{d s}{R^{2}(s)},
\end{aligned}
$$

with $R_{\tau}=\partial R / \partial \tau$ and the conditions: $\partial \mathcal{C}(0, \tau) / \partial \rho=0, \mathcal{C}(1, \tau)=0$, and $\mathcal{C}(\rho, 0)=C_{0}$. The total number of particles contained in the grain as a function of $\tau$ is obtained by transforming equation (5) to

$$
\mathcal{G}(\tau)=d \int_{0}^{1} x^{d-1} \mathcal{C}(x, \tau) d x
$$

Note that in our convention the variables are transformed to $C(r, t) \Rightarrow \mathcal{C}(\rho, \tau)$, $G(t) \Rightarrow \mathcal{G}(\tau)$ and $R(t(\tau))=\mathcal{R}(\tau)$. Solving equation (7) for $\mathcal{C}(\rho, \tau), \mathcal{G}(\tau)$ can be determined through the evaluation of the integral in (10).

Before discussing the solution of equation (7), we should note that grain growth is assumed to obey a power law (Atkinson 1988, Martin et al. 1997) according to

$$
\bar{R}(t)^{m}-\bar{R}_{0}^{m}=K t,
$$

where $\bar{R}$ is the mean grain radius and $K$ is a constant with Arrhenius temperature dependence. In the limit where $\bar{R}(t) \gg \bar{R}_{0}$, we write $\bar{R}(t)=(K t)^{\alpha}$, with $\alpha \equiv 1 / m$ being the grain growth exponent. In mean field (non-topological) theories $\alpha=1 / 2$ for both $d=2$ and $d=3$, i.e. a parabolic growth law. Grain growth experiments usually exhibit $\alpha \leq 1 / 2$, the difference could be a result of neglecting the topological constraints and the detailed microstructure.

\section{General solution}

Following the method outlined in (Forsberg \& Massih 2007), we write the general solution of equation (17) in terms of the basis components

$$
\mathcal{C}(\rho, \tau)=\sum_{i=0}^{\infty} \mathfrak{a}_{i} e_{i}(\rho, \tau)
$$


where $\mathfrak{a}_{i}$ are real numbers (constants). Next, we separate the principal time-dependence of $e_{i}(\rho, \tau)$ by making an ansatz

$$
e_{i}(\rho, \tau)=\exp \left(-\omega_{i} \tau\right) \widetilde{e}_{i}(\rho, \tau)
$$

where $\omega_{i}$ is a constant and $\widetilde{e}_{i}$ is weakly time-dependent obeying

$$
\begin{aligned}
& \frac{\partial \widetilde{e}_{i}}{\partial \tau}=\triangle_{\rho}^{\prime} \widetilde{e}_{i}+\omega_{i} \widetilde{e}_{i} \\
& \text { with } \quad \triangle_{\rho}^{\prime} \equiv \frac{\partial^{2}}{\partial \rho^{2}}+\left[\frac{d-1}{\rho}+\frac{R_{\tau}}{R} \rho\right] \frac{\partial}{\partial \rho},
\end{aligned}
$$

which is subjected to the boundary conditions: $\partial \widetilde{e}_{i}(0, \tau) / \partial \rho=0$ and $\widetilde{e}_{i}(1, \tau)=0$.

We now express the function $\widetilde{e}_{i}$ in terms of an infinite series

$$
\widetilde{e}_{i}=\sum_{k=0}^{\infty}{ }_{i} \lambda_{k} \widehat{e}_{i}
$$

where ${ }_{i} \lambda_{k} ; i \geq 1$, with ${ }_{i} \lambda_{0}=1$, and $\omega_{i}$ are selected such that the imposed boundary conditions are satisfied for all $\tau$ and ${ }_{k} \widehat{e}_{i}$ satisfy equation (14) with solutions expressed in binomial series in the form:

$$
{ }_{k} \widehat{e}_{i}(\rho, \tau)=\sum_{m=0}^{k}\left(\begin{array}{c}
k \\
m
\end{array}\right)(2 \tau)^{k-m}{ }_{i}^{m} f(\rho),
$$

where ${ }_{i}^{m} f$ satisfy the following differential equations

$$
\begin{aligned}
& \triangle_{\rho}^{\prime}{ }_{i}^{0} f+\omega_{i}{ }_{i}^{0} f=0 ; \\
& \triangle_{\rho}^{\prime}{ }_{i}^{m} f+\omega_{i}{ }_{i}^{m} f=2 m_{i}^{m-1} f ; \quad \text { for } m \geq 1
\end{aligned}
$$

with ${ }_{i}^{m} f(1)=0,{ }_{i}^{m} f(0)=\delta_{m 0}$ and ${ }_{i}^{m} f_{\rho}(0)=0 ; \delta_{m 0}$ the Kronecker delta and $f_{\rho}=d f / d \rho$. As will be shown below, ${ }_{i}^{0} f$ are expressible in terms of the confluent hypergeometric functions, whereas ${ }_{i}^{m} f$ with $m \geq 1$ are related to such functions.

Writing equation (18) in a spherical grain, we obtain

$$
\frac{d^{2} \mathbf{f}}{d^{2} \rho}+\left(\frac{d-1}{\rho}+\mathbf{c} \rho\right) \frac{d \mathbf{f}}{d \rho}+\mathbf{w} \mathbf{f}=0
$$

subject to $\mathbf{f}(1)=0, \mathbf{f}(0)=1$ and $\mathbf{f}_{\rho}(0)=0$, where $\mathbf{f}={ }_{i}^{m} f, \mathbf{c}=\partial \ln \mathcal{R} / \partial \tau \equiv \mathcal{R}_{\tau} / \mathcal{R}$ and $\mathbf{w}=\omega_{i}$. Equation (20) can be transformed to the more familiar confluent hypergeometric equation

$$
z \frac{d^{2} \mathbf{u}}{d^{2} z}+\left(\frac{d}{2}-z\right) \frac{d \mathbf{u}}{d z}-\mathbf{a u}=0
$$

subject to $\mathbf{u}(-c / 2)=0, \mathbf{u}(0)=1$ and that $\mathbf{u}_{z}(0)$ exists, where $z=-\mathbf{c} \rho^{2} / 2, \mathbf{a}=\mathbf{w} / 2 \mathbf{c}$ and $\mathbf{u}(z) \equiv \mathbf{f}(\rho)$. We should note that equations (20) and (21) are tacitly time-dependent through c. When $\mathcal{R}_{\tau} / \mathcal{R}=$ constant, c becomes a time-independent constant and exact solutions can be derived. The assumption that $\mathbf{c}=$ constant is exact for a parabolic growth law, since by recalling equation (9), it implies $d R^{2} / d t=2 D \mathbf{c}$. 
A solution of equation (21) is the confluent hypergeometric function (Dennery \& Krzywicki 1995) written in the form

$$
\mathbf{u}(z)=\Phi\left(\mathbf{a}, \frac{d}{2} ; z\right)
$$

where $\Phi(a, b ; z)$ is the Kummer function; also with alternative notations $M(a, b, z)$ or ${ }_{1} F_{1}(a, b, z)$, see e.g., (Abramowitz \& Stegun 1964). This function is regular at $z=0$ and can be expressed as a power series:

$$
\Phi(a, b ; z)=\sum_{s=0}^{\infty} \frac{\Gamma(a+s) \Gamma(b) z^{s}}{\Gamma(a) \Gamma(b+s) \Gamma(1+s)} .
$$

\section{Special solutions}

A point worth noting is that equation (21) can be expressed as an eigenvalue problem of the form $\mathcal{L} u(z)=a_{n} u(z)$, where $a_{n}$ is the energy eigenvalue of the operator $\mathcal{L}=z d^{2} / d z^{2}+(d / 2-z) d / d z$. We "quantize" the eigenvalues: $a_{n}=n+1 / 2$, where $n=0,1,2, \ldots(n \in \mathbb{N})$ and thus write

$$
{ }_{n} u(z)=\Phi\left(n+\frac{1}{2}, \frac{d}{2} ; z\right)
$$

or alternatively

$$
{ }_{n}^{0} f(y)=\Phi\left(n+\frac{1}{2}, \frac{d}{2} ;-y^{2}\right) .
$$

Here the choice of $a_{n}=n+1 / 2$ is merely to obtain a series of convenient expressions (harmonics) for the solutions ${ }_{n}^{0} f(y)$ and integrals of ${ }_{n}^{1} f(y)$, see below. For the ground state, $n=0$ and $d=3$, we have

$$
{ }_{0}^{0} f(y)=\frac{\sqrt{\pi}}{2 y} \operatorname{erf}(y),
$$

where $y=(c / 2)^{1 / 2} \rho$ and $\operatorname{erf}(y)$ is the usual error function.

Next, returning to equation (19) and writing it in the spherical system,

$$
{ }_{i}^{m} f_{\rho \rho}+\left(\frac{d-1}{\rho}+c \rho\right){ }_{i}^{m} f_{\rho}+\omega_{i}{ }_{i}^{m} f=2 m_{i}^{m-1} f ; \quad \text { for } m \geq 1
$$

Again, replacing $z=-c \rho^{2} / 2$, equation (27) is transformed to

$$
z{ }_{n}^{m} u_{z z}+\left(m+\frac{1}{2}-z\right){ }_{n}^{m} u_{z}-\left(n+\frac{1}{2}\right){ }_{n}^{m} u=-\frac{m}{c}{ }_{n}^{m-1} u ; \quad \text { for } m \geq 1
$$

where the aforementioned quantized energy eigenvalues, $\omega / 2 c=n+1 / 2$, were used, and in addition, the spatial dimension was quantized, in the manner, $d=2 m+1$. Let us write (28) for $m=1$ and $n \in \mathbb{N}$ with the convention ${ }_{n} u \equiv{ }_{n}^{1} u$, namely

$$
z_{n} u_{z z}+\left(\frac{3}{2}-z\right){ }_{n} u_{z}-\left(n+\frac{1}{2}\right){ }_{n} u=-\frac{{ }_{n} p(z)}{c} e^{z} \text {. }
$$


In writing the right-hand side of equation (29), we have utilized Kummer's relation

$$
\begin{aligned}
& \Phi\left(n+\frac{1}{2}, m+\frac{1}{2} ; z\right)=e^{z}{ }_{n}^{m} p(z), \\
& { }_{n}^{m} p(z)=\Phi\left(m-n, m+\frac{1}{2} ;-z\right),
\end{aligned}
$$

where ${ }_{n}^{m} p(z)$ is a polynomial when $n \geq m$. In particular, with ${ }_{n} p={ }_{n}^{1} p$, we have

$$
\begin{aligned}
& { }_{1} p(z)=1 \\
& { }_{2} p(z)=1+\frac{2 z}{3}, \\
& { }_{3} p(z)=1+\frac{4 z}{3}+\frac{4 z^{2}}{15}, \\
& { }_{4} p(z)=1+2 z+\frac{4 z^{2}}{5}+\frac{8 z^{3}}{105},
\end{aligned}
$$

Let us now remove $e^{z}$ from equation (29) by making the substitution ${ }_{n} u(z)=e^{z}{ }_{n} v(z)$, we obtain

$$
z_{n} v_{z z}+\left(\frac{3}{2}+z\right){ }_{n} v_{z}-(n-1){ }_{n} v=-\frac{{ }_{n} p(z)}{c} .
$$

This can be differentiated $n-1$ times to give

$$
z_{n} v^{(n+1)}+\left(n+\frac{1}{2}+z\right){ }_{n} v^{(n)}=-\frac{2^{n-1}(n-1) !}{(2 n-1) ! ! c},
$$

where the superscript in the parenthesis on ${ }_{n} v$ denotes the number of differentiations with respect to the argument. We find ${ }_{n} v$ by first integrating (37) once, which yields

$$
{ }_{n} v^{(n)}(z)=-\frac{2^{n-1}(n-1) !}{(2 n-1) ! ! c} z^{-(n+1 / 2)} e^{-z} \int_{0}^{z} \zeta^{n-1 / 2} e^{\zeta} d \zeta
$$

then with further integrations, $n$ times, we obtain

$$
{ }_{n} v(z)=-\frac{2^{n-1}}{(2 n-1) ! ! c} \int_{0}^{z}(z-s)^{n-1} \int_{0}^{s}\left(\frac{\zeta}{s}\right)^{n-1 / 2} e^{\zeta-s} d \zeta d s .
$$

After some rearrangement, we write

$$
{ }_{n} v(z)=-\frac{2^{n-1}}{(2 n-1) ! ! c} \int_{0}^{1}(1-\kappa)^{n-1 / 2} e^{-\kappa z} \int_{0}^{z} s^{n-1} e^{\kappa s} d s d \kappa .
$$

The integral over the variable $z$ can be expressed in terms of gamma functions, viz.

$$
\int_{0}^{z} s^{n} e^{\kappa s} d s=(-\kappa)^{-n-1}[\Gamma(n+1,-\kappa z)-n \Gamma(n)]
$$

where $\Gamma(a, x)$ is an incomplete gamma function (Abramowitz \& Stegun 1964). Substituting this result in equation (40) and making use of the properties of gamma functions (Abramowitz \& Stegun 1964), we finally write

$$
{ }_{n} v(z)=-\frac{2^{n-1}(n-1) !}{(2 n-1) ! ! c} \int_{0}^{1}(1-\kappa)^{n-1 / 2}(-\kappa)^{-n-2} \sum_{j=0}^{\infty} \frac{(-\kappa z)^{n+j}}{\Gamma(n+j+1)} d \kappa .
$$

Hence solution to equation (29) is found through ${ }_{n} u(z)=e^{z}{ }_{n} v(z)$. 
Let us evaluate the solution of equation (21), relation (22), when $c$ is small, i.e., $\mathcal{R}_{\tau} / \mathcal{R} \ll 1$ or $a \gg 1$. We first write $(22)$ in a quantized form

$$
\Phi\left(a, \frac{d}{2} ; z\right) \Rightarrow \Phi\left(a+m, \frac{d}{2}+m ; z\right),
$$

for $m \in \mathbb{N}$. For negative $z$, we can expand (43) in series (Abramowitz \& Stegun 1964) according to

$$
\Phi\left(a+m, \frac{d}{2}+m ; z\right)=\Gamma\left(m+\frac{d}{2}\right) e^{z / 2}\left(\frac{\sigma}{2}\right)^{\nu} \sum_{n=0}^{\infty} A_{n}\left(\frac{z}{\sigma}\right)^{n} J_{n-\nu}(\sigma),
$$

where $\sigma=\sqrt{(d-2 m) z-4 a z}, \nu=1-m-d / 2, A_{0}=1, A_{1}=0, A_{2}=d / 4+m / 2$, and

$$
(n+1) A_{n+1}=(n+d / 2+m-1) A_{n-1}+(2 a+m-d / 2) A_{n-2} .
$$

In equation (44), $J_{p}(\sigma)$ is the Bessel function of the first kind, related to the spherical Bessel function via $j_{p}(\sigma)=\sqrt{\pi / 2 \sigma} J_{p+1 / 2}(\sigma)$. Specializing to $d=3$, equation (44) can be expressed as

$$
\Phi\left(a+m, \frac{3}{2}+m ; z\right)=(2 m+1) ! ! e^{z / 2} \sum_{n=0}^{\infty} A_{n} z^{n} \sigma^{-(m+n)} j_{m+n}(\sigma),
$$

Since $A_{1}=0$, the first term in series (46) offers a good approximation for large $a$. For $m=0$, we find

$$
\Phi\left(a, \frac{3}{2} ; z\right) \approx e^{z / 2} \frac{\sin \sigma}{\sigma}
$$

with $\sigma=\sqrt{(3-4 a) z}$. Recalling the substitutions $a=\omega / 2 c$ and $z=-c \rho^{2} / 2$, we write

$$
\Phi\left(\frac{\omega}{2 c}, \frac{3}{2} ;-c \rho^{2} / 2\right) \approx \frac{e^{-c \rho^{2} / 4} \sin [\rho \sqrt{\omega-3 c / 2}]}{\rho \sqrt{\omega-3 c / 2}} .
$$

Hence for $c=0$, we have $\Phi=\sin (\rho \sqrt{\omega}) /(\rho \sqrt{\omega})$; the result which could be obtained directly from equation (22), viz.

$$
\lim _{c \rightarrow 0} \Phi\left(\frac{\omega}{2 c}, \frac{d}{2} ;-c \rho^{2} / 2\right)={ }_{0} F_{1}\left(; \frac{d}{2} ;-\omega \rho^{2} / 4\right) .
$$

The ${ }_{0} F_{1}$ function has the series expansion ${ }_{0} F_{1}(; b ; z)=\sum_{k=0}^{\infty}(\Gamma(b) / \Gamma(b+k)) z^{k} / k$ !, and can be expressed in terms of the Bessel functions of the first kind, e.g., ${ }_{0} F_{1}\left(; 3 / 2 ;-\omega \rho^{2} / 4\right)=$ $j_{0}(\rho \sqrt{\omega})=\sin (\rho \sqrt{\omega}) /(\rho \sqrt{\omega})$.

Let us now be more explicit and write the series solution from equations (13) and (16) as follows

$$
e_{i}(\rho, \tau)=\left({ }_{0} \widehat{e}_{i}+{ }_{i} \lambda_{11} \widehat{e}_{i}+{ }_{i} \lambda_{2}{ }_{2} \widehat{e}_{i}+\cdots\right) \exp \left(-\omega_{i} \tau\right),
$$

where, according to equation (17), we write

$$
\begin{aligned}
{ }_{0} \widehat{e}_{i} & ={ }_{i}^{0} f(\rho) \\
{ }_{1} \widehat{e}_{i} & ={ }_{i}^{1} f(\rho)+2 \tau{ }_{i}^{0} f(\rho) \\
{ }_{2} \widehat{e}_{i} & ={ }_{i}^{2} f(\rho)+4 \tau{ }_{i}^{1} f(\rho)+4 \tau^{2}{ }_{i}^{0} f(\rho)
\end{aligned}
$$

etc. 
The functions ${ }_{i}^{m} f$ satisfy the differential equations (18)-(19) or (27). For $c=0$, i.e. when $\left(d R^{2} / d t\right) / D \ll 1$, we have $(d=3)$

$$
\begin{aligned}
& { }_{i}^{0} f(\rho)=j_{0}\left(\rho \mu_{i}\right) \\
& { }_{i}^{m} f(\rho)=\mu_{i}^{-2 m}\left(\rho \mu_{i}\right)^{m} j_{m}\left(\rho \mu_{i}\right),
\end{aligned}
$$

where $\mu_{i}=\sqrt{\omega_{i}}$. Satisfying the boundary condition ${ }_{i}^{0} f(1)=0$, we obtain

$$
\mu_{j}=j \pi \quad j=0,1,2, \ldots
$$

Next, we calculate the the total number of particles per unit volume of the grain at "time" $\tau$; i.e. equation (10), expressed as

$$
\mathcal{G}(\tau)=\sum_{i=0}^{\infty} \mathcal{G}_{i}(\tau)
$$

where using equation (12) and in three dimensions, we write

$$
\mathcal{G}_{i}(\tau)=3 \int_{0}^{1} x^{2} e_{i}(x, \tau) d x .
$$

Substituting for $e_{i}(x, \tau)$ from equation (50) and employing the relations in (51) gives

$$
\begin{aligned}
\mathcal{G}_{i}(\tau)= & 3 \int_{0}^{1} x^{2}\left[{ }_{i}^{0} f(x)+{ }_{i} \lambda_{1}\left({ }_{i}^{1} f(x)+2 \tau_{i}^{0} f(x)\right)+\right. \\
& \left.+{ }_{i} \lambda_{2}\left({ }_{i}^{2} f(x)+4 \tau{ }_{i}^{1} f(x)+4 \tau^{2}{ }_{i}^{0} f(x)\right)+\cdots\right] e^{-\mu_{i}^{2} \tau} d x .
\end{aligned}
$$

The terms in equation (57) can be integrated by using the relation:

$$
\int_{0}^{1} x^{2}{ }_{i}^{n} f(x) d x=\mu_{i}^{-1} j_{n+1}\left(\mu_{i}\right)
$$

In a numerical treatment the parameters ${ }_{i} \lambda_{1},{ }_{i} \lambda_{2}$ are determined by satisfying the boundary conditions at the beginning and the end of each discretized time interval. Moreover, the coefficients $\mathfrak{a}_{i}$ in equation (12) are determined from the the spatial initial

condition $\mathcal{C}(\rho, 0)=C_{0}$. Assuming the orthonormality of the solutions, then in general

$$
\mathfrak{a}_{i}=\frac{\int_{0}^{1} \mathcal{C}(\rho, 0) e_{i}(\rho, 0) \rho^{d-1} d \rho}{\int_{0}^{1} e_{i}(\rho, 0)^{2} \rho^{d-1} d \rho} .
$$

\section{Evaluation}

Let us now evaluate the time variation of the number of particles contained in the grain (survival rate), viz.

$$
\frac{d S}{d t}=K_{d} \frac{\partial}{\partial t} \int_{0}^{R(t)} r^{d-1} C(r, t) d r,
$$


where $K_{d}=2 \pi^{d / 2} / \Gamma(d / 2)$. Differentiating under the integral and using equations (2) and (1), we obtain

$$
\frac{d S}{d t}=K_{d} D R(t)^{d-1}\left[\frac{\partial C(r, t)}{\partial r}\right]_{r=R(t)} .
$$

We can also calculate the time derivative of $G(t)$, expressed in the form

$$
\dot{G}(t)=d\left[\frac{\dot{S}(t)}{K_{d} R(t)^{d}}-\frac{\dot{R(t)}}{R(t)} G(t)\right],
$$

where over-dot denotes temporal differentiation. Substituting for $\dot{S}$ from equation (61) and simplifying, we find

$$
\dot{G}(t)=d\left[\frac{D}{R}\left(\frac{\partial C}{\partial r}\right)_{R}-\frac{\dot{R}}{R} G(t)\right] .
$$

This equation shows the balance between particle diffusion and grain growth. If we designate the characteristic frequency of particle diffusion by $\nu_{D}=D / R^{2}$ and that of grain growth by $\nu_{G}=\dot{R} / R$, we notice that when $\nu_{D} \ll \nu_{G}$ the grain growth is dominating and particles cannot reach the boundary of the cell and $G=C_{0}\left(R_{0} / R\right)^{d}$ with $R_{0}$ denoting the initial cell size. Using the relation for the grain growth, $R=(K t)^{\alpha}$, we can write

$$
G(t) \sim t^{-\alpha d} \text { for } \quad \nu_{G} \gg \nu_{D}
$$

For example, in 3-dimensional spherical grain with $\alpha=1 / 2, G(t) \sim t^{-3 / 2}$. Thus the total number of particles in the grain remains constant with time, i.e., $S=V(t) G(t)=$ const.

On the other hand when $\nu_{D} \gg \nu_{G}$, the diffusion flux is the controlling parameter for escape rate. It may be illustrating to evaluate $\dot{G}(t)$ in this regime for $d=3$ when $R$ is stationary, with the well-known exact solution (Carslaw \& Jaeger 1959)

$$
\begin{aligned}
\dot{G}(t) & =3 \frac{D}{R}\left(\frac{\partial C}{\partial r}\right)_{R} \text { for } \quad \nu_{G} \ll 1 \\
G(\mathcal{T}) & =6 C_{0} \sum_{k=1}^{\infty} \frac{\exp \left(-k^{2} \pi^{2} \mathcal{T}\right)}{k^{2} \pi^{2}} .
\end{aligned}
$$

Here $\mathcal{T}=D t / R^{2}$ and also equation (65) gives $G(0)=C_{0}$, as it should. In the long-time limit only the slowest decaying eigenmode contributes; hence the asymptotic number of particles per unit volume decays according to

$$
G(\mathcal{T}) \sim C_{0} e^{-\pi^{2} \mathcal{T}} \quad \text { for } \quad \nu_{G} \ll 1 \wedge \mathcal{T} \gg 1
$$

The corresponding formula for particle survival in the grain is

$$
S(t) \sim(K t)^{3 \alpha} e^{-\pi^{2} D K^{-2 \alpha} t^{1-2 \alpha}} \text { for } \nu_{G} \ll 1 \wedge D t / R^{2} \gg 1,
$$

where again we employed $R=(K t)^{\alpha}$. Hence for $\alpha<2, S(t)$ exponentially decays to zero at long times.

Let us finally investigate the case of a slowly growing grain by means of the adiabatic approximation (Krapivsky \& Redner 1996). In this method, the asymptotic solution is constructed to have the same functional form as in the stationary grain, but in 
addition to satisfy the time-dependent boundary condition, namely, $C(R(t), t)=0$ and $C(r, 0)=C_{0}$. We write $(d=3)$

$$
C(r, t) \approx \frac{R(t)}{r} \cos \left(\frac{\pi r}{2 R(t)}\right) f(t) \equiv C_{a d}(r, t),
$$

where the function $f(t)$ is determined by substituting equation (68) into the diffusion equation (11), which yields

$$
\dot{f}(t)=-\frac{\pi^{2} D+4 R \dot{R}}{4 R^{2}}\left[1+\frac{2 \pi r \dot{R}}{\pi^{2} D+4 R \dot{R}} \tan \left(\frac{\pi r}{2 R}\right)\right] f(t) .
$$

Now by placing $R=(K t)^{\alpha}$, with $\alpha<1 / 2$, into equation (69) the second term in the square bracket is negligible and we write

$$
\frac{d f}{d t} \approx-\left(\frac{\pi^{2} D}{4 R^{2}}+\frac{\dot{R}}{R}\right) f
$$

If we further consider that $\nu_{D} \gg \nu_{G}$, then $f(t)$ is given by

$$
f(t) \approx \exp \left[-\frac{\pi^{2} D}{4(1-2 \alpha) K^{2 \alpha}} t^{1-2 \alpha}\right]
$$

which is the same result obtained by Krapivsky \& Redner (1996) for the one-dimensional case. The corresponding relation for $S(t)$ is

$$
S(t) \approx K^{3 \alpha} t^{3 \alpha} \exp \left[-\frac{\pi^{2} D}{4(1-2 \alpha) K^{2 \alpha}} t^{1-2 \alpha}\right]
$$

which is consistent with equation (67).

\section{Summary}

In this note we have treated the problem of particles undergoing Brownian motion in an expanding sphere whose surface absorb the particles (Dirichlet condition). The time-dependent diffusion equation for particles in $d$-dimensional sphere, which accounts explicitly for its expansion is formulated. The equation is solved by separating the space and time variables and the spatial equation for the particle density is reduced to an ordinary confluent hypergeometric differential equation, which implicitly contains the grain growth process. For a parabolic grain growth law analytical solutions in terms of special functions are derived. When grain growth is dominant over particle diffusion, the number of particles per unit volume contained in the grain is calculated to evolve with time as $G(t) \sim t^{-\alpha d}$, where $\alpha$ is the growth exponent and $d$ the spatial dimensionality. For the case of slow growth, i.e. when the ratio of grain growth rate to diffusivity is small an asymptotic solution is attained. In this regime the long-time limit gives $G(t) \sim \exp \left[-\pi^{2}\left(D / K^{2 \alpha}\right) t^{1-2 \alpha}\right]$, where $D$ is the particle diffusivity and $K$ the growth constant. The paper has focused on the mathematical and physical aspects of the problem, numerical evaluations for a similar kind of problem are treated elsewhere (Forsberg \& Massih 2007). 
Diffusion of particles in an expanding sphere with an absorbing boundary

\section{References}

Abramowitz M \& Stegun I A 1964 Handbook of Mathematical Functions Dover New York.

Atkinson H V 1988 Acta Metallurgica 36, 469-491.

Bray A J \& Smith R 2007 a Journal of Physics A: Mathematical and Theoretical 40, 10965-10972.

Bray A J \& Smith R 2007b Journal of Physics A: Mathematical and Theoretical 40, F235-F241.

Carslaw H S \& Jaeger 1959 Conduction of Heat in Solids Oxford University Press Oxford, United Kingdom. Section 9.3.

Dennery P \& Krzywicki A 1995 Mathematics for Physicists Dover Publications Mineola, New York.

Forsberg K \& Massih A R 2007 Modelling and Simulation in Materials Science and Technology 15, 335353.

Gadomski A, Luczka J \& Rudnicki R 2003 Physica A 325, 284-291.

Ham F S 1958 Journal of Physics and Chemistry of Solids 6, 335-351.

Hargreaves R \& Collins D A 1976 Journal of British Nuclear Energy Society 15, 311-318.

Krapivsky P L \& Redner S 1996 American Journal of Physics 64, 546-552.

Martin J W, Doherty R D \& Cantor B 1997 Stability of Microstructure in Metallic Systems Cambridge University Press Cambridge, United Kingdom.

Niemiec M, Gadomski A, Luczka J \& Schimansky-Geier L 1998 Physica A 248, 365-378.

Redner S 2001 A Guide to First-Passage Processes Cambridge University Press Cambridge, United Kingdom.

Speight M V 1969 Nuclear Science and Engineering 37, 180-185.

Thompson C V 2001 Solid State Physics Vol. 55 Academic Press. 